\title{
GLAD!
}

Revue sur le langage, le genre, les sexualités

$07 \mid 2019$

Varia

\section{Archi-écritures de genre et politiques de différance : immondices verbales et littéracies d'intervention dans le quotidien des établissements scolaires}

Gender Writing and Politics of Différance: Verbal Filth and Intervention

Literacies in Schools Daily Life

\section{Rodrigo Borba et Adriana Carvalho Lopes}

Traducteur : Clara Domingues

\section{CpenEdition}

Journals

Édition électronique

URL : http://journals.openedition.org/glad/1754

DOI : $10.4000 /$ glad. 1754

ISSN : 2551-0819

Éditeur

Association GSL

Référence électronique

Rodrigo Borba et Adriana Carvalho Lopes, «Archi-écritures de genre et politiques de différance: immondices verbales et littéracies d'intervention dans le quotidien des établissements scolaires ", GLAD! [En ligne], 07 | 2019, mis en ligne le 05 décembre 2019, consulté le 17 décembre 2020. URL : http://journals.openedition.org/glad/1754 ; DOI : https://doi.org/10.4000/glad.1754

Ce document a été généré automatiquement le 17 décembre 2020.

\section{(i)

La revue GLAD! est mise à disposition selon les termes de la Licence Creative Commons Attribution Pas d'Utilisation Commerciale - Pas de Modification 4.0 International. 


\title{
Archi-écritures de genre et politiques de différance : immondices verbales et littéracies d'intervention dans le quotidien des établissements scolaires
}

\author{
Gender Writing and Politics of Différance: Verbal Filth and Intervention \\ Literacies in Schools Daily Life
}

\section{Rodrigo Borba et Adriana Carvalho Lopes}

Traduction : Clara Domingues

\section{RÉFÉRENCE}

Référence : Borba R. \& Carvalho Lopes A., Escrituras de gênero e políticas de différance : Imundície verbal e letramentos de intervenção no cotidiano escolar, Linguagem e Ensino, (Brésil) vol. 21, 2018. URL : https://periodicos.ufpel.edu.br/ojs2/index.php/rle/article/ view/15198

La revue Linguagem e Ensino publie en accès libre des textes scientifiques évalués par des pairs. Son site précise que la mise en accès libre, immédiat et gratuit des connaissances scientifiques produites vise à contribuer à une meilleure démocratisation des connaissances au niveau mondial. Cette politique prévaut également pour les traductions. Ainsi, la revue GLAD! a obtenu à titre gracieux le droit de traduire cet article et de le diffuser selon les principes que nos deux revues partagent et promeuvent : en accès libre et gratuit, avec mention de la source. 


\section{Introduction}

1 En 2016, à l'occasion de son élection à la présidence du Tribunal suprême fédéral, la ministre Cármen Lúcia fut interrogée par Ricardo Lewandowski sur la manière dont il conviendrait de l'appeler. Dans un sourire indéniablement fielleux, elle lui répondit: «J'ai fait des études et j'ai l'amour de la langue portugaise. Par conséquent, je pense que le titre de la fonction est "presidente"3. » Aussitôt après, un autre ministre affirma qu'elle serait une presidenta inocenta ("présidente innocente ») ${ }^{4}$, ce qui fit rire tout le monde. Le positionnement linguistique de Cármen Lúcia retentissait comme une réponse aux discours de Dilma Rousseff qui, dès sa prise de pouvoir en tant que chef de l'exécutif en 2011, se proclama presidenta ("présidente») du Brésil. Ce détail apparemment trivial de la langue portugaise, le genre grammatical, devint alors le centre de débats enflammés. Des spécialistes et des profanes entretinrent de longues disputes sur l'usage «correct» du terme. Des linguistes, déployant moult détails techniques et objectivité scientifique, affirmaient que presidenta faisait partie du lexique de la langue et que le terme était donc correct bien qu'inhabituel. Les discours profanes, eux, révélaient des questions plus profondes quant à la relation entre langue et politique.

2 Celles et ceux qui rejetaient cette désinence féminine parlaient au nom de la défense du bon portugais et ne faisaient aucun mystère de leur opposition politique au gouvernement modérément de gauche de Rousseff : « Il veut détruire notre langue tout comme il veut détruire notre pays. » En face, celles et ceux qui se mirent à utiliser le terme presidenta affirmaient qu'il était nécessaire de souligner que, pour la première fois, le Brésil était gouverné par une femme, soutenant ainsi que les changements dans la structure sociale modifiaient la structure de la langue ${ }^{5}$.

3 Malgré les quiproquos provoqués par l'apparition du terme presidenta, d'un point de vue linguistique, il n'y avait là aucune innovation. L'amour débridé de la ministre Cármen Lúcia et de tant d'autres Brésiliennxs ${ }^{6}$ pour la langue portugaise les rendait, pour ainsi dire, aveugles : cet item lexical existe depuis au moins un siècle, lorsque Machado de Assis en fit usage dans Mémoires posthumes de Brás Cubas. Il est entré dans les dictionnaires au XIX ${ }^{e}$ siècle pour désigner " l'épouse du président ", mais également «celle qui préside» (Houaiss 2001). Et au niveau législatif, Juscelino Kubitscheck promulgua, en 1956, la loi 2 749/1956 qui impose le genre grammatical féminin pour les titres de fonctions publiques assumées par des femmes. La nouveauté linguistique qui engendra tellement d'émoi sur le juste et le faux en portugais et de jugements sur les capacités intellectuelles de la présidente Dilma Rousseff n'en était donc pas une. L'inouï était, en revanche, qu'une femme occupât une fonction qui avait toujours été masculine, ce qui conféra au terme un air de nouveauté. Le litige autour de presidenta était ainsi motivé par du purisme et de la misogynie, cette dernière étant structurante dans la société brésilienne. Pour autant, le genre, en tant que catégorie, n'est que rarement pris en considération dans l'examen de la conjoncture politique du pays (Borba 2017).

4 L'affaire du terme presidenta illustre le fait que le genre grammatical, phénomène linguistique peu problématisé dans les analyses tant prescriptives que descriptives du portugais, est si naturalisé que tout écart dans son usage, toute fissure dans le système de la langue, se voit sanctionné par des railleries. Les débats acharnés sur le terme 
presidenta fournissent un bon exemple des questions étudiées dans cet article : (1) les innovations de genre grammatical et (2) les discours et actions qui les entourent et (in)forment (sur) leurs usages. Par «innovations de genre grammatical», nous entendons les modifications qui touchent à la structure de la langue et sèment, d'une certaine manière, la pagaille dans le système de genre - considéré comme stable et arbitraire -, avec le double objectif de miner la dichotomie de genre social, le sexisme dans la langue, et d'éviter le masculin générique. Ces innovations découlent en soi d'un élan politique qui tend à supprimer les inégalités de genre dans la langue et la société. Certaines de ces innovations incluent, entre autres, l'usage de structures disjonctives pour le syntagme nominal (par exemple : tous et toutes ; étudiants/étudiantes ; enseignant/ es, etc.), de l'arobase en remplacement de la désinence de genre pour les substantifs et les adjectifs (par exemple : tout@s les étudiant@s), de la lettre x comme effacement du genre grammatical (par exemple: toutxs les éudiantxs), d'une lettre non marquée entrainant une générification grammaticale, en portugais le $e$ (par exemple, todes alunes pour todos alunos e todas alunas) ${ }^{7}$. Nous discuterons ici, plus particulièrement, de l'usage du $x$ et d'autres interventions sémiotiques dans des contextes scolaires. Nous examinerons les discours que l'effacement du genre grammatical engendra dans les médias et les institutions d'enseignement, et ce faisant nous analyserons le rapport entre langue, société, citoyenneté et littéracie.

\section{Classé $X$ : purisme versus déstabilisation de la structure linguistique}

5 Comme le constate Kira Hall (2007), le $x$ possède un long pedigree sémiotique. René Descartes l'introduisit dans la pensée mathématique pour parler de quantités indéterminées. Plus tard, au XVII ${ }^{\mathrm{e}}$ siècle, dans les domaines de la littérature et de la loi, cette lettre fut utilisée pour nommer des personnes inconnues ou préserver l'anonymat. Dans les arts plastiques, la figure lascive de Madame X, portrait réalisé par John Singer Sargent, revêtit la lettre d'une teinte de démesure sexuelle. Durant le XX siècle, des comités de censure, aux États-Unis, utilisèrent le $x$ pour classifier des films inappropriés à un jeune public: les films classés X. Dans les années 1990, l'industrie pornographique raviva cette pratique et adopta respectivement les classes XX et XXX pour les films pornographiques hardcore et ultrahardcore. Hall remarque aussi que, plus récemment, la lettre " a acquis une nouvelle vie en tant que symbole de provocation, sinon de menace, relativement à des choses devant rester sous surveillance" (Hall $2007:$ 1562).

6 Ainsi, le $x$ condense en soi diverses couches signifiantes qui semblent, comme nous le verrons, se transposer dans le domaine de la langue: dans certains cercles, cette intervention linguistique est apparentée à un acte quasi pornographique souillant la langue et devant être censuré. À l'instar des films pornographiques, de tels usages sont classés X. Malgré les discours prohibitionnistes, le $x$ persiste dans différents contextes en tant que forme extrême de négation de la dichotomie de genre. En ce sens, l'usage $\mathrm{du} x$ par des collectifs féministes et $\mathrm{LGBTQI}^{8}$, tout comme les discours sur cet usage révèlent que ce que nous entendons par langue constitue un terrain mouvant, sans points d'appui fixes, un phénomène qui abonde en zones d'indétermination entourées de pratiques de surveillance et de sanction. 
7 Si même le genre grammatical - qui a toujours été considéré comme nécessaire à la langue, car il serait le reflet incontestable de la biologie - traverse des zones de turbulence, rien ne garantit plus la stabilité tant désirée du social. Or, nous le verrons plus loin, en désessentialisant le système, de telles propositions de changement linguistique provoquent une insécurité sociale qui se matérialise dans des pratiques visant à surveiller la pureté de la langue. Dans notre époque de grands changements sociaux, les certitudes se diluent. Pour reprendre Zygmunt Bauman (2010 [2003]), tout ce qui semblait solide s'est effondré. Tout ce qui semblait absolu se révèle relatif. Tout ce qui pouvait faire l'objet de jugements catégoriques tels que « juste » ou « faux » s'est évaporé. "Tout, sauf la langue» (Faraco 2013: 266). Dans ce contexte, la langue apparait comme l'ultime bastion des certitudes. Interdit d'y toucher. C'est à partir de cette perspective que nous argumentons ici que la langue et surtout ce que nous en faisons et ce que nous en disons ne peuvent être considérés comme une question secondaire dès lors que nous prétendons appréhender des moments historiques de turbulence sociale et politique.

\section{Phonophallogocentrisme et (dés)invention du portugais}

8 Le 22 septembre 2015, le journal O Globo fit savoir que des enseignantxs du Colégio Pedro II, à Rio de Janeiro, avaient adopté le $x$ pour se référer aux élèves sans distinction de genre ${ }^{9}$. L'article reproduisait des copies d'examen et des affichettes collées sur les murs de l'établissement dans lesquelles était utilisée, plutôt que le masculin générique (convention adoptée pour se référer à des groupes mixtes), une lettre ne supportant aucune désinence de genre, ainsi que nous le voyons ci-dessous.

Figure 1

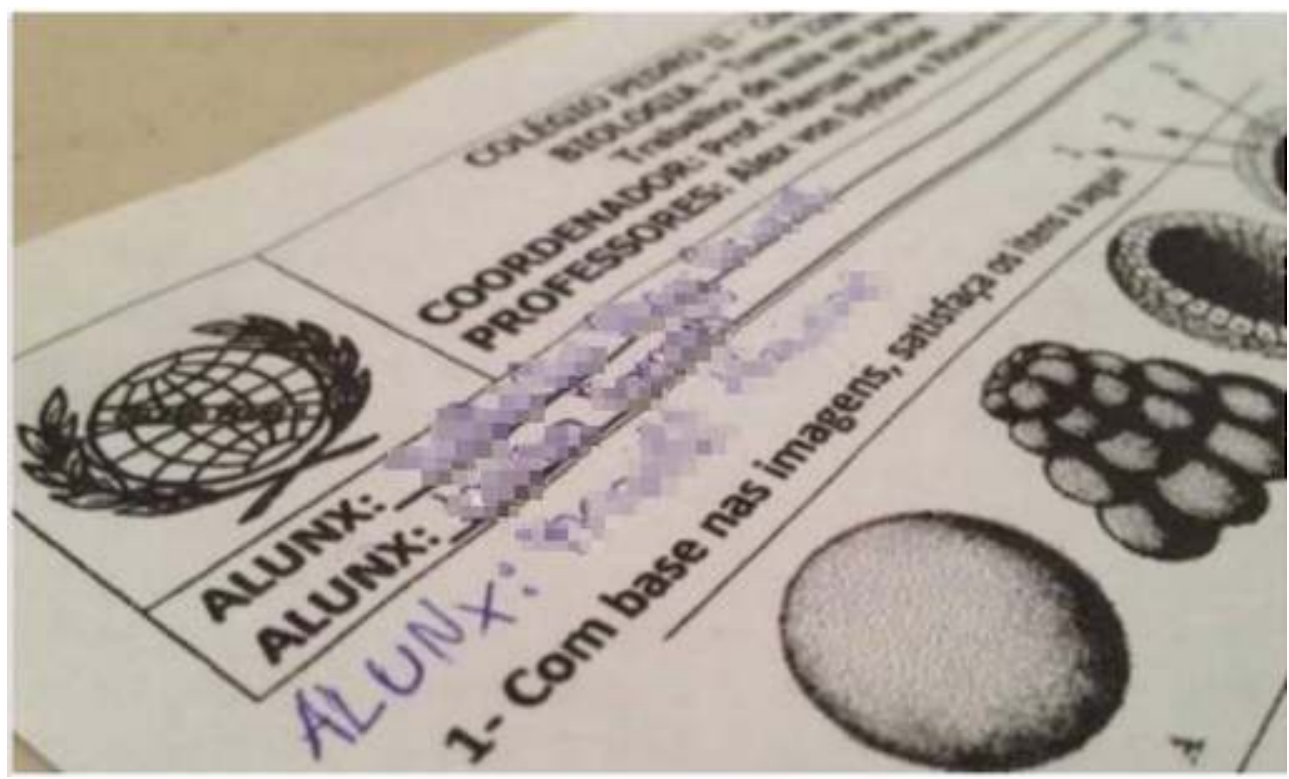




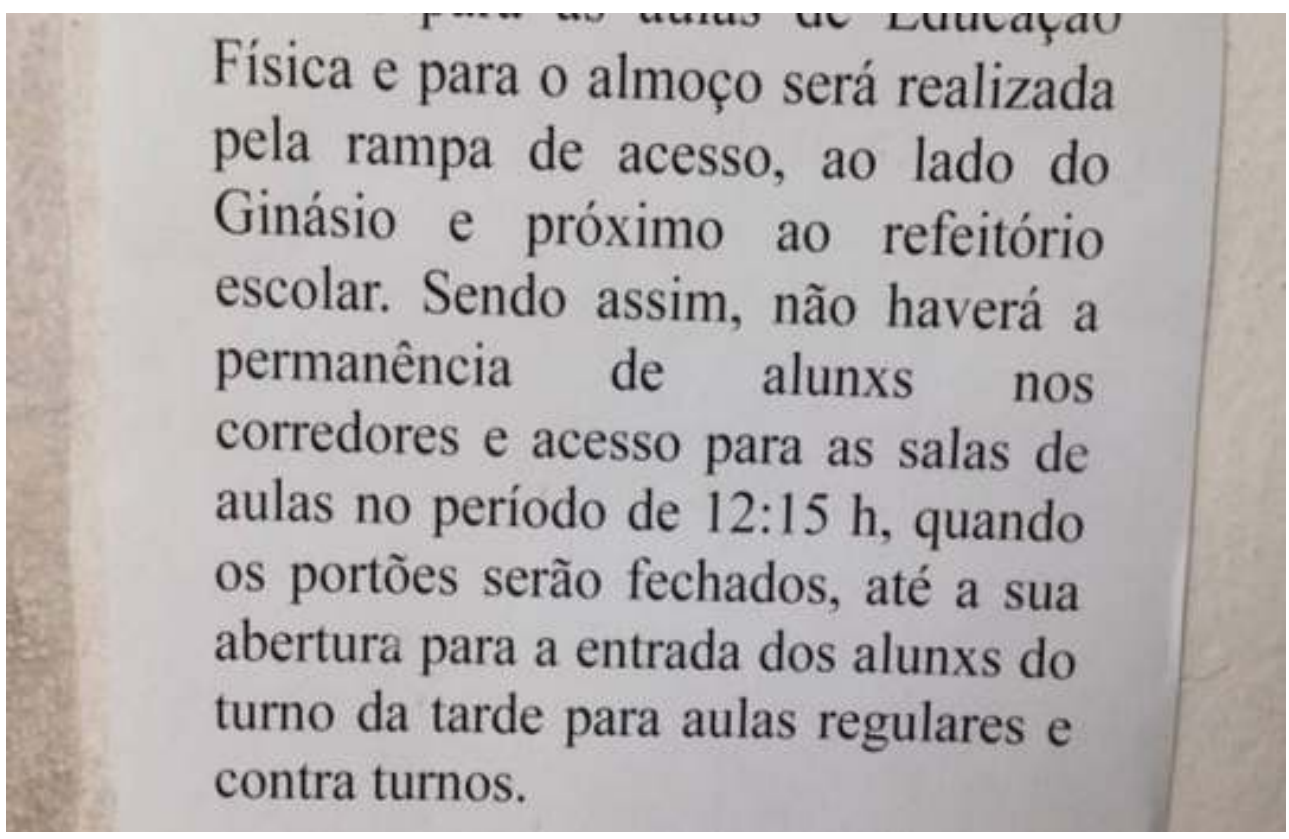

nouvelle provoqua, bien sûr, l'émoi parmi les spécialistes et les usagerxs ordinaires du langage. En effet, comment l'une des institutions publiques d'enseignement les plus traditionnelles du pays, qui établit en 1898 la grammaire nationale en tant qu'objet d'étude et dont les enseignantxs conçurent les premières grammaires scolaires (Soares 2012), pouvait-elle prendre la tête d'une telle ineptie linguistique? Évidemment, le $x$ et autres innovations de genre grammatical n'ont pas été inventées par les enseignantxs du Colégio Pedro II. Des mouvements sociaux féministes et LGBTQI s'intéressent depuis des décennies à la manière dont la langue peut être excluante et oppressive lorsque, par sa soumission à la règle du masculin générique, elle gomme certaines expériences de vie. Ces mouvements voient la langue comme un instrument important dans la lutte pour la libération, contre l'oppression et l'invisibilisation (Cameron 1992 ; Caldas-Coulthard 2007). Au Colégio Pedro II, c'est le syndicat étudiant qui introduisit le $x$ dans ses journaux et supports d'information. Sensibiliséxs par l'intérêt de certainxs élèves à l'égard des possibilités politiques de la langue, des enseignantxs mirent en place des activités interdisciplinaires sur la question, exercice qui trouva son point culminant dans l'en-tête de la copie d'examen et l'affichette reproduites ci-dessus (figures 1 et 2 ).

La nouveauté ne résidait donc pas dans l'usage du $x$. Ce qui causa tant de frissons, ce fut son apparition dans un environnement scolaire dont on attendait une standardisation de la norme dite savante. La publication d'une telle information occasionna dès lors d'innombrables pratiques de régulation de la langue, que nous analyserons plus loin. Dans l'immédiat, il importe d'observer que cette querelle linguistique (Cameron 2012) sur le genre grammatical révèle que, bien au-delà d'une structure et d'un système abstrait de signes, la langue constitue un projet discursif (Woolard 1998) sur lequel s'abattent des implications politiques, morales et sociales qui visent à réguler sa matérialisation et son usage.

11 Dans ce contexte, nous adoptons la perspective actuellement développée par les études sur le langage et leurs théorisations sur la manière dont la langue telle que nous l'entendons (ce qui la constitue et ses fonctions sociales) n'est pas circonscrite au 
système linguistique per se, mais à ce que les usagerxs pensent que leur langue devrait être et comment elle devrait fonctionner (Woolard 1998; Bauman \& Briggs 2003; Kroskrity 2004 ; Moita Lopes 2013 ; Cameron 2012, 2014). Les langues nous offrent, en effet, les moyens de parler et d'écrire, mais elles nous fournissent aussi de la matière pour parler et écrire sur elles. Pour parler portugais (ou français, anglais, guarani, swahili, etc.), il faut préalablement l'inventer, laisser se répandre certains régimes de vérité (Foucault 1980) qui, avant toute autre chose, affirment que cette langue existe et ce qu'elle doit être (Pinto 2013). En d'autres termes, les discours sur la langue forgent des connaissances déterminées sur elle et (in)forment (sur) les rapports que nous entretenons avec la ou les langue(s) que nous parlons. Cette tâche discursive est entreprise tant par des linguistes que par (et peut-être surtout) des usagerxs ordinaires dans leur vie quotidienne, les unxs et les autres parlant et écrivant abondamment sur la langue.

De même que l'emploi du substantif presidenta, les autres interventions concernant le genre grammatical suscitent de grands émois. Aussi, nous posons ici la question de savoir comment et pourquoi l'usage du $x$ au Colégio Pedro II a pu engendrer tant d'attaques discursives. À ce sujet, Deborah Cameron nous explique que toute modification dans la structure linguistique s'accompagne en général de pratiques d'hygiène verbale, définies par l'autrice comme :

un ensemble hétérogène de discours et de pratiques à travers lesquelles des personnes essaient de «nettoyer » la langue et de faire en sorte que sa structure ou ses usages se conforment à leurs idéaux de beauté, de vérité, d'efficacité, de logique, de correction et de civilité. [Cet ensemble] n'est pas une entreprise futile motivée par l'incapacité à comprendre comment fonctionne la langue. Au contraire, l'hygiène verbale est un produit de la manière dont la langue fonctionne: elle résulte de notre capacité réflexive et métalinguistique qui confère à la communication son caractère flexible et nuancé. (Cameron 2012 : vii, c'est l'autrice qui souligne)

Les discours hygiénistes sur la langue ne surviennent pas dans un contexte de vide social. À l'inverse, ils découlent d'une certaine idéologie linguistique formée par ce que Jacques Derrida (1967) a appelé l'épistémè phonologocentrique et ce que Donna Haraway (2009 [1991]) a qualifié de phallogocentrisme. En suivant Derrida et Haraway, nous comprenons que les angoisses mises en lumière par l'usage du $x$ comme marque du genre sont causées par la menace que ces usages linguistiques font peser sur ce que nous pourrions appeler le phonophallogocentrisme, i.e. une conception philosophique et scientifique (mais aussi une conception relevant largement du sens commun) qui fait de la langue un simple miroir, une "représentation » d'une réalité ou d'une vérité ontologique antérieure et extérieure à elle ${ }^{10}$. En d'autres termes, cette perception sert de base à l'idée selon laquelle il y aurait une " chose en tant que telle », une "réalité en tant que telle", une ontologie existant indépendamment de ce que nous disons à son sujet. Une réalité pure. Cameron (1990) souligne que l'un des mythes constitutifs non seulement de la linguistique, mais de la sociolinguistique elle-même (la branche de cette science qui aspire à réunir l'étude sur la langue et la société) serait que la langue entretient un rapport spéculaire à la réalité, autrement dit que la langue ne fait que refléter la réalité. Si dans le monde réel nous vivons de manière dichotomique en tant qu'hommes et que femmes, comment la langue pourrait-elle se soustraire à une telle catégorisation? 

perspective spéculaire quant au rapport langue/réalité et rendre manifestes les limites de cette épistémè phonophallogocentrique. En effet, toucher à la langue, c'est toujours toucher à quelque chose de plus. Nos préoccupations linguistiques rejoignent indiscutablement des questions non linguistiques, elles constituent et expriment des positionnements qui se révèlent à l'occasion de changements sociaux. En d'autres termes, la réflexivité métalinguistique est intimement liée à des questions sociales, morales et politiques qui forgent des régimes de vérité déterminés sur certaines questions : comment est la langue? comment elle doit fonctionner? quel est son rapport à la réalité ?

Dans le cas du $x$, cette réflexivité se voit exacerbée par le fait que celui-ci n'affecte que la langue écrite. Il n'a pas à être prononcé ${ }^{11}$. Sa radicalité n'est pas uniquement sociale ; elle est structurelle. Dans les sociétés graphocentriques, où l'écrit tient une place centrale, la surveillance institutionnelle exercée par les organes normalisateurs (dictionnaires, académies des lettres, écoles, universités, livres didactiques, etc.) sur l'écrit est accrue. Dès lors, nous pourrions imaginer que le graphocentrisme subvertit ce que nous qualifions de régime de vérité phonophallogocentrique dans lequel l'écrit est entendu comme un simple dérivé de la langue orale - qui, elle, serait à valoriser philosophiquement et scientifiquement, puisque seule la phonie aurait la capacité de refléter une vérité non linguistique transcendantale.

Cependant, un tel raisonnement se révèle quelque peu hâtif. Comme nous le verrons plus loin, parmi les arguments défendant la tradition au sein de la langue écrite, il n'y a, en réalité, aucune inversion qui ébranlerait la dichotomie oral/écrit. La vénération de l'écrit dans nos sociétés n'interroge pas et ne dénaturalise pas les présupposés constitutifs d'un tel binarisme qui continue à dissimuler une violente hiérarchie. En d'autres termes, l'écrit ne cesse jamais d'être vu comme le dérivé d'un sens original et auto-identique d'un être ou d'une essence non linguistique préexistant à la condition de l'être en question. Nul hasard si, dans des métadiscours de linguistes et de grammairiens ${ }^{12}$, le fait de modifier l'écriture du genre grammatical n'implique pas une transformation du genre social, puisque le premier est considéré comme détenteur d'une supposée vérité prédiscursive qui émane de la Voix et ne fait qu'être transposée ou reflétée dans l'écrit. Contrariant le principe même du caractère arbitraire de la langue, postulé par Saussure, l'écrit serait alors la «représentation (écrite)» de la «représentation (orale)» de l'origine (être), ou encore le «signifiant (gramma)» du « signifiant (phoné) » du signifié. Nous pouvons donc considérer ces métadiscours sur le genre grammatical comme des «instruments politiques de structuration du champ social (hiérarchisation et légitimation d'un certain ordre sociolinguistique)" (Signorini 2012 : 110) et de la langue. Et étudier ces discours sur la langue peut nous aider à comprendre comment certains régimes de vérité la constituent et, par conséquent, comment des usagerxs de cette langue affrontent et (ré)organisent le social.

\section{Archi-écritures de genre: des immondices verbales}

À l'instar d'autres langues romanes, le portugais marque le genre des substantifs, des pronoms, des articles, des adjectifs, des participes passés et des gérondifs substantivés. Il possède ainsi un genre lexical, morphologique et grammatical. Malgré 
l'omniprésence de cette catégorie linguistique, rares sont les études systématiques sur le genre grammatical en portugais. Le savoir disponible provient, en général, d'analyses linguistiques proposées par des grammaires qui problématisent peu le rapport entre genre grammatical et genre social, construisant de la sorte un régime de vérité phonophallogocentrique dans lequel ce rapport est postulé comme naturel et inévitable. Cela n'est guère surprenant, en réalité, dans la mesure où la linguistique tend à mettre le social entre parenthèses (Bourdieu 1977; Bagno 2013) pour s'assurer une prétendue objectivité scientifique. Mattoso Câmara (1975:77) argumente ainsi que "le caractère masculin ou féminin d'un mot est immanent au mot et de nature lexicale» et non sociale. En ce sens, le fait que le portugais (comme d'autres langues) possède un masculin générique (i.e. l'usage du masculin pour se référer à l'humanité comme un tout) est considéré comme un simple phénomène linguistique et non comme le produit de conventions sociales et politiques élaborées dans des sociétés patriarcales.

En anglais, par exemple, une étude diachronique, désormais classique, d'Ann Bodine (1975) montre que le masculin générique résulte de l'institutionnalisation des grammaires prescriptives entre les XVII ${ }^{e}$ et $\mathrm{XVIII}^{\mathrm{e}}$ siècles. Élaborées par des hommes (les femmes étant exclues de la sphère de la connaissance jusqu'alors), ces grammaires imposèrent le genre masculin comme métonymie de l'humanité. L'argument de Bodine fut si accablant qu'il occasionna une pléiade d'études. Ensemble, celles-ci finirent par forger ce qu'on appelle aujourd'hui la linguistique féministe (Penelope 1982; Cameron 1992 ; Pauwels 1998; Abbou 2011; Abbou \& Baider 2016). Dans les contextes anglo-saxons, la critique féministe de la langue produisit, de fait, des résultats concrets : des manuels d'écriture non sexiste furent publiés (Miller \& Swift 2002) et des organes normalisateurs, comme des universités et des dictionnaires, recommandent aujourd'hui d'éviter des structures clairement sexistes telles que l'usage du masculin générique. Évidemment, ces changements linguistiques ne survinrent pas sans avoir préalablement été la cible de discours fortement opposés à ces interventions féministes (Penelope 1982 ; Cameron 2012).

Bien que le féminisme brésilien soit un mouvement social et académique vigoureux, Joana Pinto et Suzana Badan (2012) suggèrent, après avoir passé en revue 20 années de publications féministes sur le portugais du Brésil, que les préoccupations de nos linguistes féministes se concentrèrent sur le discours et négligèrent de problématiser le sexisme qui se matérialise au niveau du système. L'étude de Carmen Rosa CaldasCoulthard (2007) constitue, à cet égard, une exception. Selon cette autrice, en portugais, le masculin générique est attendu dans des textes qui se réfèrent à un groupe mixte de personnes (comme, par exemple, l'affichette sur le mur du Colégio Pedro II) et toute innovation dans cet usage (par exemple, le $x$ ) suscite un sentiment d'étrangeté et engendre des discours hygiénistes visant à défendre le caractère non marqué du masculin. Dans ce contexte, Caldas-Coulthard (2007: 234) argumente que, même si le genre grammatical semble être un simple détail linguistique, une recherche qui allierait analyses au niveau systémique et discursif embrasserait des questions sociopolitiques bien plus larges. En d'autres termes, tout comme le genre social est marqué par des relations de pouvoir, le genre grammatical est traversé par des asymétries qui débordent le linguistique et touchent au social, et inversement.

On ne peut comprendre l'affaire du Colégio Pedro II, d'un point de vue analytique, si l'on ne prend pas en compte cet entrelacement entre le niveau systémique de la langue et les discours qui régissent et (ré)inventent ce système, autrement dit les pratiques 
d'hygiène verbale que ces discours entrainent. Au niveau systémique, le $x$ ne se contente pas de défier l'institutionnalisation du masculin générique. Son audace consiste à perturber la norme, à la faire plier sur elle-même et à désessentialiser un aspect linguistique fortement naturalisé ; dans une perspective derridienne, le $x$ efface la relation spéculaire entre réalité, oralité et écrit, et défie la primauté de l'épistémè phonophallogocentrique, que celle-ci résulte de la linguistique ou du sens commun. Au niveau discursif, en déstabilisant la prétendue naturalité du genre (grammatical et social), cette nouveauté engendre des débats enflammés lors desquels sont produits des discours susceptibles de nous en apprendre beaucoup sur notre rapport non seulement à la langue, mais surtout aux changements sociaux qui nous environnent.

21 Loin de nettoyer la langue, d'en assurer l'hygiène, ce changement au niveau du système, parce que politiquement motivé, la souille. En contrepoint de ce que Cameron a appelé "hygiène verbale ", nous concevons le $x$ et d'autres innovations relatives au genre grammatical comme des formes d'«immondices verbales" qui défient le phonophallogocentrisme, en poussant la langue hors de son espace de pureté et en la contaminant par des usages mondains, politiques et éthiques. C'est la radicalité structurelle et sociale de ces innovations linguistiques qui nous amène à les considérer comme des formes d'immondices. En effet, elles ne prétendent pas nettoyer la langue, mais bien y mettre la pagaille, elles défient le purisme linguistique, brouillent les binarismes propres au niveau systémique (oralité/écrit; signifiant/signifié, etc.), contaminent la langue par un agenda politique et la sortent ainsi de sa zone de confort aseptique.

Deux dimensions signifiantes traversent métapragmatiquement ces innovations linguistiques. La première concerne les idéologies linguistiques (Kroskrity 2004 ; Moita Lopes 2013) qui les perçoivent comme une forme de souillure, une faute de soin et de tenue par rapport à l'ordre établi, comme une source de chaos devant être éradiquée pour que la langue (et la société) demeure propre et stérilisée tant linguistiquement que politiquement. Idéologies entretenues et perpétuées par les « amoureusxs » du bon portugais. Il n'est pas inutile de rappeler, ainsi que le souligne Susan Gal (1995), que les idéologies linguistiques se restreignent rarement à la sphère du langage. En effet, ce qui les rend analytiquement performantes comme forme de critique sociale, c'est qu'elles " sont systématiquement liées à d'autres domaines tels que la nature des personnes, du pouvoir et d'un ordre moral souhaitable» (Gal 1995 : 171). Les querelles motivées par l'article sur l'usage du $x$ au Colégio Pedro II illustrent bien la manière dont l'idéologie linguistique voit dans cette innovation une souillure indésirable. La tentative de protéger la pureté de la langue survient dès l'instant où est émis le jugement selon lequel il existerait un relâchement moral de la part de l'établissement et des enseignantxs qui soutiendraient ainsi la dégénérescence intellectuelle et politique des élèves.

23 Le 23 septembre 2015 (à $3 \mathrm{~h}$ du matin !), le journaliste Reinaldo Azevedo publie, sur le blog (désormais fermé) qu'il tient pour le magazine Veja, ce qu'il pense de l'affaire du Colégio Pedro II. ${ }^{13}$ 


\section{Patrulha gayzista e feminazi chega ao Colégio Pedro II, uma instituição federal}

Une patrouille gayziste et féminazie débarque au Colégio Pedro II, institution fédérale publié la veille par le journal $O$ Globo. Azevedo semble y dénoncer le fait que l'institution aurait été envahie par des groupes de tendance fasciste, dictatoriale et violente, comme en témoignent clairement les néologismes gayziste et féminazi qui appartiennent, on le comprend aisément, au champ sémantique du nazisme. L'établissement se serait-il donc transformé en un camp de concentration, pour ainsi dire, contrôlé par des gays et des féministes cruellxs ? Nous ne découvrons le motif de l'inquiétude d'Azevedo que dans le corps du texte. Il y explique avec consternation: genre grammatical et la décadence de la langue, mais les mouvements sociaux qui interfèrent avec le système sous un motif politique. Pour ce commentateur, le $x$ est source de nausée et, dans sa tentative de nettoyer la langue (et l'école) de ces immondices, il jette le discrédit sur des individus qui ont lutté, historiquement, pour une société plus égalitaire. La réflexivité métalinguistique d'Azevedo, en se référant à la langue, se réfère plus encore à celles et ceux qui l'utilisent. Les commentaires du journaliste ne laissent aucun doute sur le fait que sa préoccupation principale ne porte pas sur l'intégrité linguistique, mais sur une supposée probité morale de l'établissement qui serait, selon lui, en pleine décomposition. En d'autres termes, en discourant sur la langue, Azevedo se montre davantage inquiet de la «nature des personnes, du pouvoir et d'un ordre moral souhaitable» (Gal 1995 : 171). Pour lui, les immondices verbales (et morales) sont le signe de changements sociaux qu'il abomine.

Cameron (2012) observe que les tentatives de protéger la langue contre les souillures du monde apparaissent aussi dans des discours de spécialistes. Le point commun à tous ces discours réside dans la distinction radicale entre le linguistique et le social. On y défend la langue en l'installant, pour ainsi dire, dans une tour d'ivoire et en l'éloignant de ce que les usagerxs en font et en pensent. Tel est le positionnement que développent les linguistes Gisela Collischonn et Luiz Carlos Schwindt dans un texte publié par le journal Zero Hora, le 12 décembre 2015, et ayant pour sous-titre : « pourquoi la distinction entre genre social et grammatical est nécessaire à la langue $»^{14}$. D'après ces enseignantxs, en 
portugais, le genre grammatical n'est pas nécessairement lié à la catégorie sémantique du sexe. En effet, pour des mots tels que bolo (" gâteau ») ou casa (« maison »), le genre grammatical est inhérent au mot, il possède un caractère lexical, non social. Collischonn et Schwindt expliquent aussi qu'en portugais, il n'y a convergence entre genre grammatical et social que pour $13 \%$ des substantifs, par exemple pour les mots $o$ engenheiro ("l'ingénieur») ou a advogada ("l'avocate»). Quant au masculin générique, ces linguistes assurent que : "Tous" inclut hommes et femmes; “toutes" n'inclut que les femmes. Dans de nombreuses langues, le genre non marqué est le masculin, ce qui ne signifie pas un rapport de pouvoir de l'homme sur la femme.» Voilà la base analytique de leur principal argument; pour ces linguistes, comme le titre de leur article l'indique clairement, « ceci est ceci, cela est cela ». Pour le dire plus clairement, ceci est la langue et ses catégories inhérentes et nécessaires; cela est la vie sociale et ses changements irrépressibles.

Une telle analyse ignore les travaux de linguistes féministes comme Bodine (1975), Penelope (1982), Pauwels (1998) et Abbou (2011) qui ont précisément problématisé que certaines conventions linguistiques ne sont pas naturelles à la langue, mais bien le fruit de conventions et de négociations politiques. Ainsi que le démontre la recherche diachronique très détaillée de Bodine (1975), le caractère non marqué du masculin ne fut institutionnalisé en anglais qu'entre les $\mathrm{XVII}^{e}$ et $\mathrm{XVIII}^{\mathrm{e}}$ siècles. Jusque là, dans différents contextes, le masculin générique cohabitait (presque) harmonieusement avec l'usage du pronom à la troisième personne du pluriel they associé à un référent singulier ${ }^{15}$. Autrement dit, bien que, pour certains substantifs (tels que bolo (" gâteau ») et casa («maison »)), le genre grammatical ne relève effectivement pas d'une question de pouvoir, quand il s'agit de distinguer le genre social, le genre grammatical sert de catalyseur aux inégalités. Dans leur article, Collischonn et Schwindt tentent de tracer des frontières très claires entre langue et société, comme si l'une ne dépendait pas de l'autre, comme si l'une ne rétroalimentait pas l'autre. Selon ces spécialistes, les innovations relatives au genre grammatical ignorent des questions chères à la linguistique. Nous posons, quant à nous, la question suivante : qu'en est-il des questions chères à qui utilise la langue et perçoit dans cet usage des relations de pouvoir inégalitaires?

Le mot immondice vient du latin imundus ("sale »), lui-même formé du préfixe négatif in- et du substantif mundus ("propre» ou "monde »). Par conséquent, la deuxième dimension signifiante qui traverse ces innovations de genre grammatical les envisage comme une manière de faire sortir la langue de sa zone de confort, de défier son statu quo, de la souiller, pour ainsi dire, par des intérêts politiques spécifiques et de la faire descendre du piédestal où elle est érigée en système abstrait de signes, en l'insérant dans le monde, in mundus. Autrement dit, si, dans le monde actuel, le genre n'est pas une pratique aussi dichotomique et binaire que les discours essentialistes et l'écriture normalisée des grammaires le proclament, biffer le genre grammatical d'un $x$ ou à l'aide d'autres stratégies implique de salir la langue de toute l'instabilité et la fluidité du social. Rendre la langue immonde, c'est donc la placer dans le monde, là même où elle peut contribuer au changement social. Telle est l'idéologie portée par des groupes qui luttent contre les oppressions de genre et sexuelles, comme les militantxs féministes et LGBTQI. 
convient ici de souligner les propos d'Oscar Halac, proviseur du Colégio Pedro II, lorsqu'il réfute les critiques sur l'usage du $x$ au sein de son institution. Dans un entretien accordé au journal $O$ Globo, il affirme :

Au Colégio Pedro II, nous nous positionnons en faveur du respect de l'être humain. Rien ne sert de former un expert en mathématique ou en portugais si l'on ne forme pas aussi un citoyen. Insérer les élèves dans une société citoyenne devrait être un engagement pris par tous les établissements scolaires ${ }^{16}$.

Peut-être plus important encore pour notre propos est le positionnement d'une élève de Pedro II, qu'elle développe dans un entretien non dirigé avec le premier auteur de cet article :

À l'école, il y a des femmes, des hommes, des pédés, des lesbiennes, des trans, des hétéros, des bis. Il y a des gens qui disent être des deux genres, d'autres qui n'en ont aucun. Il y a un petit peu de tout. Alors il n'y a pas de raison de parler uniquement d'étudiants, parce qu'il n'y a pas que des étudiants. Le collectif féministe du CPII utilise le $\mathrm{x}$ depuis longtemps déjà parce que nous pensons qu'il est plus inclusif, il est non genré, chacune l'entend à sa manière, il est plus ouvert, il laisse le genre en suspens. Je suis fière que mon établissement utilise le $\mathrm{x}$ parce que le CPII a toujours été très traditionnel, mais aujourd'hui il fait savoir qu'il commence à se préoccuper de la réalité de l'école, des privilèges dont bénéficient certaines personnes, des problèmes quotidiens dont on souffre parce qu'on vit dans une société machiste telle que la nôtre. Que ce soit juste ou faux, peu m'importe. Ce qui m'importe, c'est que ça fait une différence.

31 Ce témoignage se situe à l'extrême opposé de ce que pensent le journaliste et les linguistes précédemment mentionnéxs. L'élève met ici en évidence que langue et société ne peuvent être comprises comme des strates indépendantes de notre existence. Elle laisse, en effet, entrevoir que « le genre, compris comme une catégorie grammaticale, ne peut être pensé indépendamment de son contexte culturel » (Abbou \& Baider $2016: 5$ ). Étant donné qu'à l'école, il y a « des femmes, des hommes, des pédés, des lesbiennes, des trans, des hétéros, des bis ", la distinction de genre grammatical ne rend pas compte, selon elle, du fait qu'à Pedro II il y a « un petit peu de tout ». Cette élève place ainsi le social au cœur du linguistique ; elle place la langue dans le monde, in mundus, et la souille de ses motivations politiques, morales et éthiques.

Comme nous l'indiquons dans le titre de cet article, nous considérons ces immondices verbales moins comme une écriture que comme une archi-écriture, qui non contente de renverser la dichotomie oral/écrit - établie par la société graphocentrique questionne aussi les prémisses du phonophallogocentrisme. À ce point du raisonnement, il convient de discuter un peu de la distinction entre écriture et archiécriture suggérée par Derrida. Alors que la première est une technique entendue comme une "image", un "travestissement" d'une langue originelle (telle que conçue de Platon à Saussure), l'archi-écriture est un principe de différenciation sans origine.

Dans son projet de déconstruction, Derrida (1967) argumente que ce qui caractérise l'écriture se trouve aussi dans la parole. L'absence d'une présence - des interlocuteurxs dans une conversation et de la "chose même» dont on parle, par exemple - n'est pas seulement une caractéristique de l'écriture, mais un principe général du langage. Il en est ainsi parce que la notion de langue chez Derrida est traitée 
de manière quelque peu différente que chez Saussure. Pour le précurseur de la linguistique moderne, la langue est un système abstrait synchroniquement présent, dans lequel un élément prend sens dans son opposition à un autre élément absent et est représenté par la parole. De plus, Saussure élabore son concept de langue en excluant l'écriture. En effet, celle-ci serait extérieure [à la linguistique], secondaire, d'où son rôle purement technique par rapport à la parole, qu'il lui revient de re-présenter.

Introduisant dans la discussion précisément ce que Saussure avait exclu de sa Linguistique générale (l'écriture), Derrida (tout comme les enseignantxs et l'élève de Pedro II) comprend la langue comme une machine à produire de la différance, néologisme qui réécrit le mot différence sans en modifier la prononciation et implique que le signifié advient précisément dans le rapport entre différence et ajournement du sens. Le a de différance déstabilise la primauté de la parole et, par-dessus tout, montre inévitablement qu'aucun élément ne peut être re-présenté ; un tel élément serait à peine un simulacre, une promesse jamais tenue, ou encore, une trace. De sorte qu'il n'existerait pas de signifié pur et indépendant ni de signifiant en tant que chose concrète. Un signifiant se rapporte toujours à un autre signifiant, puisqu'il n'existe pas de signifié transcendantal, aprioristique - de chose «en tant que telle». Tout sens s'établirait à travers une chaine d'éléments dans laquelle un concept se rapporte à un autre dans un jeu de différences. Il n'y a qu'articulations entre eux, autrement dit le sens se constitue dans un réseau de différance et de trace. Cette chaine nous amène à ce que Derrida appelle l'archi-écriture.

Ainsi, à nos yeux, l'archi-écriture non seulement renverse la place secondaire de l'écriture (en tant que travestissement), mais fait du travestissement lui-même et, dans notre affaire, des immondices verbales un principe non négatif : pour le langage, seul existe un mouvement référentiel infini d'un signifiant à l'autre, qui jamais ne trouve d'origine. Mais, puisqu'il n'y a pas de présence, qu'est-ce qui garantit une fixation minimale de sens de manière à ce que les mots puissent être, d'une manière ou d'une autre, lisibles et leurs sens partagés, ajoutés aux dictionnaires ? Pour Derrida (1972), ce qui assure cette lisibilité est le caractère répétable et disséminateur du langage. Aussi, le philosophe élabore deux concepts complémentaires: "itérabilité » et " citationnalité ».

Du sanscrit itara, « autre », l'itérabilité est la propriété par laquelle le signe est toujours autre dans sa mêmeté, répétition dans l'altération; autrement dit il est répétition y compris en l'absence de la personne destinataire et de ce que veut dire le ou la locutrice. Quant à la citationnalité, elle est la propriété par laquelle le signe est soustrait à son contexte « original » et déplacé dans un autre contexte et, par là même, produit du signifié (à ce sujet, voir Borba 2014). Voilà ce que serait l'une des caractéristiques générales de l'archi-écriture : elle ne reflète pas une réalité antérieure, elle n'est même pas prisonnière d'un contexte spécifique ; elle est toujours marquée par une rupture, une différance. Dans cette perspective théorique, vu qu'il n'a pas de référent originel, tout signe (soit-il phonique ou graphique) peut être greffé, cité, soustrait à son contexte et, pourtant, continuer à être lisible et à produire des effets. Derrida (1972 : 377) écrit ainsi :

Il appartient au signe d'être en droit lisible même si le moment de sa production est irrémédiablement perdu et même si je ne sais pas ce que son prétendu auteurscripteur a voulu dire en conscience et en intention au moment où il l'a écrit, c'està-dire abandonné à sa dérive essentielle. 
L'archi-écriture semble donc défier la valorisation de l'écriture, considérée par les sociétés graphocentriques comme plus solide et moins transitoire que la parole. Les institutions qui structurent les régimes hégémoniques de littéracie (comme les écoles, les universités, les tribunaux, etc.) insistent sur le fait que l'écriture, parce qu'elle bénéficie d'un support physique apparemment plus stable, connait une plus grande permanence que la parole qui est irrépréhensiblement changeante. Ce serait dans l'écriture que la conscience des interlocuteurxs se conserverait à tout jamais. Au final, comme dit le proverbe, verba volant, scripta manent (i.e. les paroles s'envolent, les écrits restent). Cependant, l'archi-écriture rend immonde la langue, elle tord radicalement le cou à une telle manière de voir, car aucun signe n'est inébranlable, aucun signe ne possède de frontière immuable, aucun signe ne reflète une parole originale et un vouloir-dire authentique. Nous prenons le risque d'avancer que, dans les sociétés graphocentriques, plus qu'une valorisation de l'écriture, il y aurait une intensification du contrôle et de la surveillance à son encontre en raison de sa caractéristique supposément stable et disséminatrice (à ce sujet, voir Weth \& Juffermans 2018). Reinaldo Azevedo et sa guerre contre les interventions linguistiques de « gayzistes » et de « féminazis » à Pedro II nous confortent dans cette hypothèse.

Pour ce qui est du fameux $x$ : en défiant l'écriture, son archi-écriture met aussi au défi la forme dichotomique et hétéronormative des genres. Une telle construction n'existe, en effet, qu'à la faveur de la conception phonophallogocentrique qui postule des vérités prédiscursives, y compris pour les corps: les formes masculine et féminine seraient alors le simple reflet d'une réalité antérieure et extérieure à la langue. Cependant, pour les personnes qui réécrivent les genres dans la langue, cette perception est renversée. Pour elles, langue et réalité sont intrinsèquement liées. Nul hasard si l'élève de Pedro II rapporte que, dans son établissement,

il y a des gens qui disent être des deux genres, d'autres qui n'en ont aucun. Il $\mathrm{y}$ a un petit peu de tout. Alors il n'y a pas de raison de parler uniquement d'étudiants, parce qu'il n'y a pas que des étudiants.

Nous pourrions dire que, pour ces usagerxs, rendre la langue immonde revient à s'immiscer dans la réalité, à souiller le social avec du linguistique et le linguistique avec du social. Dans ce scénario, peu importe de savoir si l'usage du $x$ est juste ou faux, il importe seulement que cette intervention dans le système linguistique "fasse une différence » dans la vie des gens.

\section{Le $x$ de la discorde : littéracies d'intervention et politiques de la différance}

L'usage du $x$ et d'autres immondices en contextes scolaires n'est pas une pratique exclusive du Colégio Pedro II. Le Groupe Diversité, par exemple, un collectif d'étudiantxs LGBT de l'Université Fédérale Rurale de Rio de Janeiro (UFRRJ), organisa, au deuxième semestre 2015, sa première semaine d'intégration. L'affiche ci-dessous était accrochée à l'entrée du campus. 


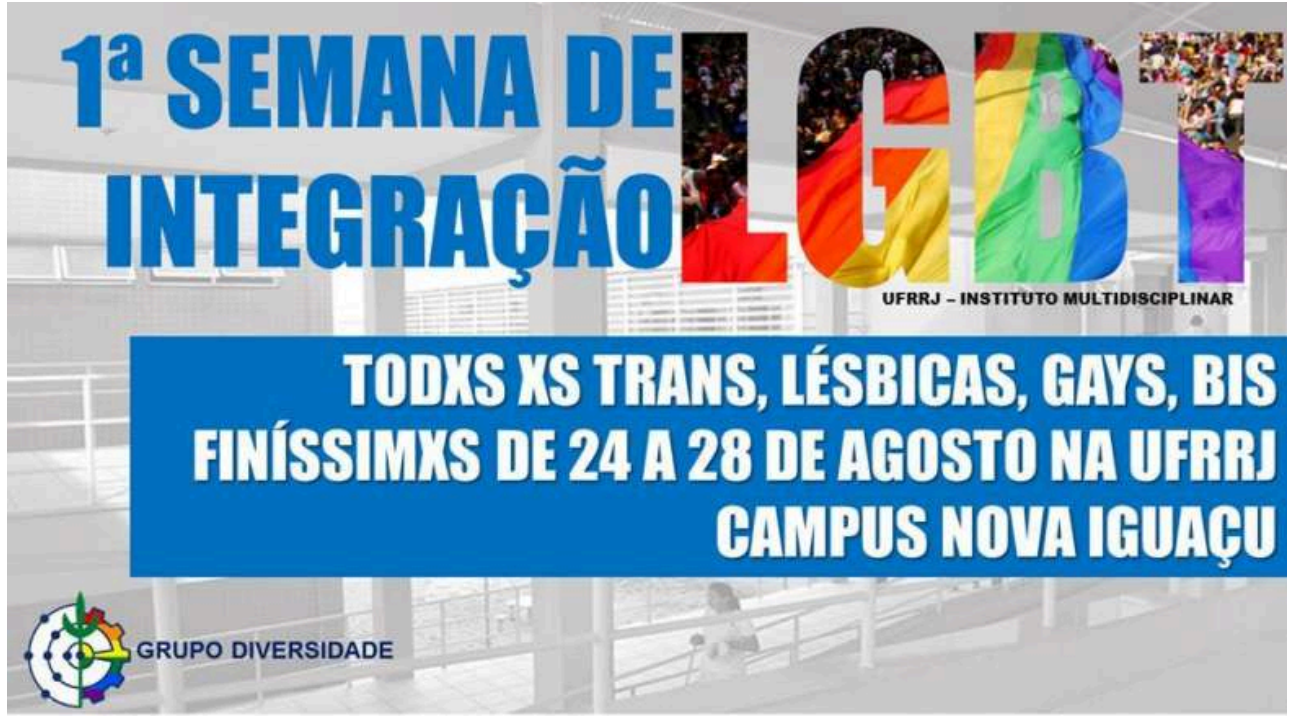

jère semaine d'intégration LGBT

UFRRJ - Institut multidisciplinaire

Trans, lesbiennes, gays, bis

Toutxs pimpantxs du 24 au 28 août à l'UFRRJ

Campus Nova Uguaçu

Groupe Diversité

Ce ne fut pas tant cette affiche, ni même les divers ateliers, conférences et événements culturels prévus cette semaine-là qui contrarièrent fortement étudiantxs, enseignantxs et personnel non enseignant. Les raisons de la discorde furent les innombrables affichettes accrochées par les collectifs sur les portes des toilettes au sein de l'institution: des écrits rendant la langue immonde, désessentialisant les genres et semblant disséminer, d'une certaine manière, la déconstruction de la langue et des corps, au-delà des espaces dévolus à l'événement. Voici deux exemples qui, à l'image du $x$, réécrivent et désessentialisent les genres. 
Figure 5

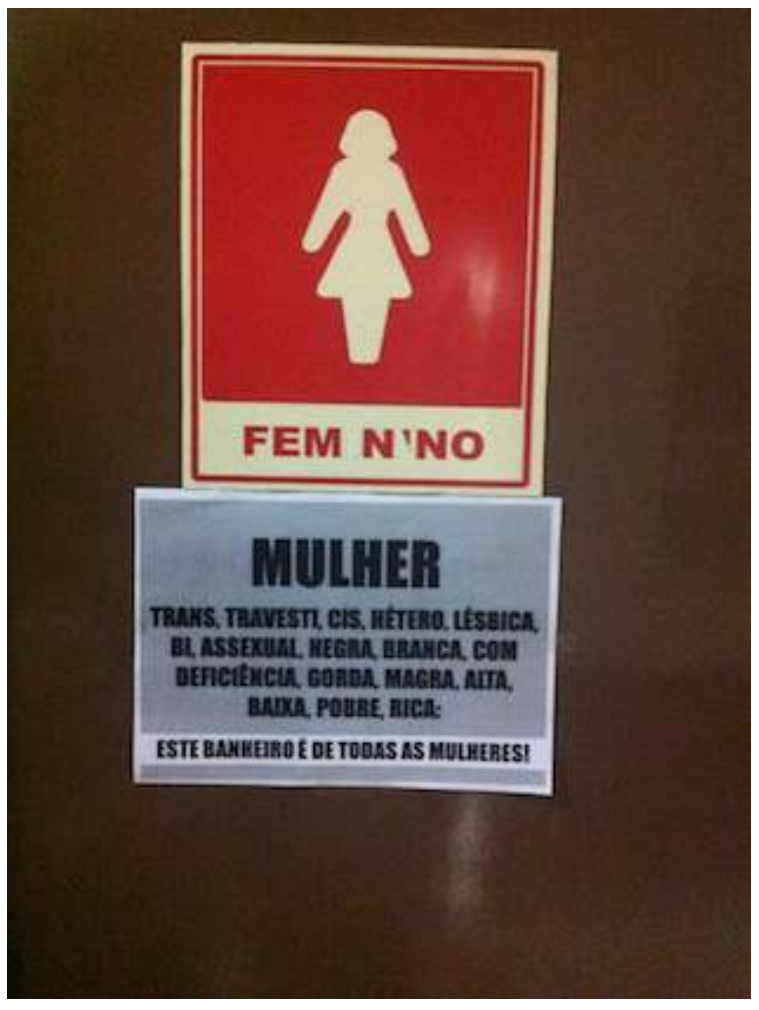

FEMME

Trans, travestie, cis, hétéro, lesbienne, bi, asexuelle, noire, blanche, avec handicap, grosse, maigre grande, petite, pauvre, riche:

ces toilettes sont pour toutes les femmes! 


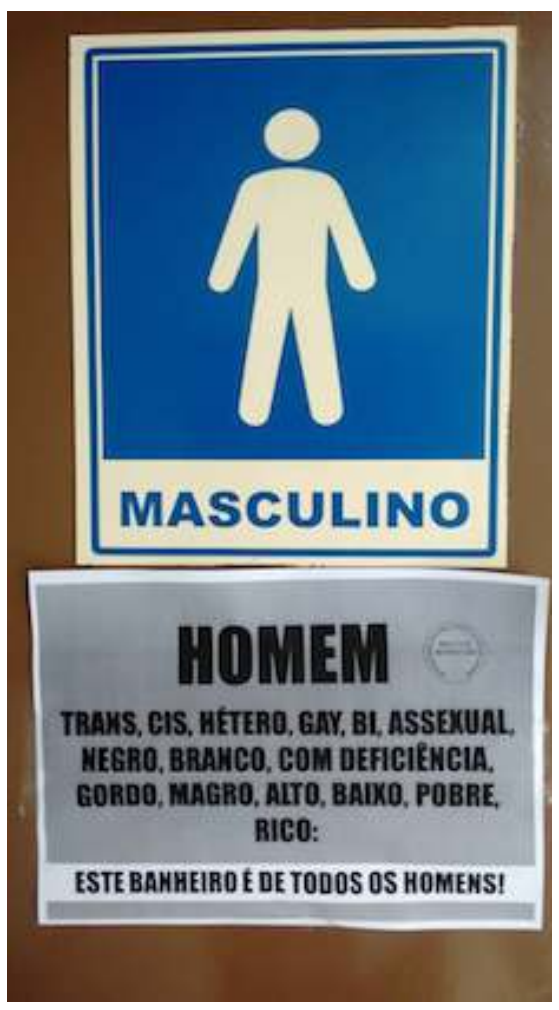

HOMME

Trans, cis, hétéro, gay, bi, asexuel, noir, blanc, avec handicap, gros, maigre, grand, petit, pauvre, riche : ces toilettes sont pour tous les hommes!

Qu'est-ce qui justifie d'accrocher des affichettes explicatives en dessous des symboles feminino («féminin ») et masculino (« masculin ») fixés aux portes des toilettes? Et puis, pourquoi ces affichettes ont-elles à ce point irrité et incommodé de nombreuses personnes? Il nous semble que, plus qu'une paraphrase cherchant à dire avec d'autres mots la même chose et, ainsi, à « éclaircir » et compléter un sens qui serait déjà là, plus qu'un mouvement qui fixe et contrôle le sens, ces affichettes opèrent le mouvement inverse. Elles déplacent le sens du symbole, elles lui retirent sa stabilité, la présence de l'original qu'il présume, le rapport supposément plus naturel entre les corps duels (signifiants) et leurs respectives représentations dichotomiques (signifiés ${ }^{17}$ ). À l'instar du $x$ dont nous avons discuté ci-dessus, ces paraphrases transforment les symboles masculin et féminin en signifiants qui glissent et se déploient en tant d'autres, des signifiants qui réécrivent, de la sorte, les innombrables manières d'être féminin et masculin, une femme et un homme : trans, cis, hétéro, gay, asexuellx, noirx, blanchx, etc. La réflexivité métapragmatique des usagerxs qui écrivent ces paraphrases troublantes montre que les symboles masculin et féminin, tout comme les termes homme et femme, ne sont pas des signifiants aussi inclusifs que la linguistique le voudrait (voir, par exemple, l'argument des linguistes dont il a précédemment été question).

C'est précisément cette caractéristique incontrôlable de la dissémination des signes et des immondices verbales qui dérangea tant au sein du campus universitaire. En guise de témoignage, un des enseignantxs qui collabora à l'organisation de l'événement avec les étudiantxs, rapporta ce qui suit sur sa page Facebook: 
les affichettes collées sur les portes des toilettes n'ont même pas tenu une semaine. La plupart ont été arrachées le jour même. Il faut rappeler que du personnel non enseignant, ainsi que des ENSEIGNANTS [sic] sont allés voir la direction pour protester contre la présence de ces affichettes, certaines personnes allant jusqu'à exiger le cachet de la direction. Tout ça pour déconsidérer l'intervention des étudiant@s et, bien sûr, la légitimité de la cause. Quelques jours plus tard, j'ai réimprimé d'autres affichettes et à nouveau elles ont toutes été arrachées en quelques heures... Bref, on a des progrès à faire sur la question. indiquer la centralité de la dimension collective et agissante de ce type de démarche. Par conséquent, les littéracies d'intervention "surviennent ", autrement dit elles viennent après quelque chose, elles réécrivent et (re)signifient une écriture antérieure et hégémonique, traditionnellement conçue comme neutre ou comme un simple reflet de la réalité. Par ailleurs, il s'agit d'une écriture qui « est entre » des personnes; elle n'est pas tissée dans la solitude, à l'image de l'écriture scolaire telle qu'elle est traditionnellement conçue, elle est, au contraire, un processus qui se construit dans le collectif et au-delà des lieux de contrôle qui lui sont dévolus. C'est de cette manière que ces interventions rendent immonde le quotidien scolaire, qu'elles placent la vie et les processus d'apprentissage moraux, politiques et éthiques in mundus. Ce type de littéracie recrée et resignifie des réalités, faisant ainsi la différance - autrement dit ces

GLAD!, 07 | 2019 
interventions réécrivent et ré-imaginent une écriture hétéronormative et phonophallogocentrique, générant, de la sorte, des plissements dans des lieux de pouvoirs institutionnalisés (i.e. école, grands médias, etc.). Comme nous l'avons démontré à travers les affaires de Pedro II et de l'UFRRJ, ces collectifs d'étudiantxs recréent des écritures, tordent l'ordre établi, rendent immonde la littéracie et donnent de la visibilité à des questions sociales et politiques qui traversent la langue, interrogeant, par là même, des arrangements de pouvoir.

\section{Considérations finales} lorsqu'elles rendent la langue immonde en contextes scolaires, témoignent de mouvements de résistance et de réarticulation des normes qui, paradoxalement, ne fonctionnement qu'en raison de leur propre inefficacité : dans la mesure où la norme n'a pas d'essence, d'origine, elle a besoin d'être continuellement réitérée, fixée et contrôlée. Le scandale soulevé par les grands médias autour de l'usage du $x$ dans un établissement considéré comme le berceau de la tradition, ainsi que la réprobation qui s'est manifestée à l'encontre de ces réécritures sur les portes des toilettes au sein d'une institution de l'enseignement supérieur révèle la puissance de cette surveillance. Cependant, nous pourrions dire, en suivant Butler (2006 [1990]), que si ces réitérations créent les dichotomies de genre qui paralysent, excluent et stéréotypent, en rendant vulnérables les gens considérés comme différents, ce sont aussi des répétitions qui échouent, qui sont à la dérive et servent de terrain à la réarticulation de signifiants mettant quotidiennement au défi les normes scolaires quant à la naturalité des genres et de la langue.

À l'image du $x$ qui apporte une part d'énigme et d'indétermination dans l'apparent rapport spéculaire entre signes phoniques et signes graphiques (comment lire le $x$ ?) et ébranle la logique phonophallogocentrique, d'autres interventions sur le genre dans la langue biffent la manière binaire d'enseigner/apprendre. Comme le soulignent Lima et Borba, à l'école, apprendre signifie, traditionnellement, «se laisser saisir par le "ou", accepter ses termes et agir dans les limites qu'il établit: (...) tu es un blanc ou un noir, un homme ou une femme, un riche ou un pauvre?»(2016:80). Nous pourrions poursuivre ces chaines dichotomiques ad infinitum, mais comme elles échouent, nous pouvons aussi les interrompre, montrer toute la violence qui les a constituées. En ce sens, les littéracies d'intervention nous indiquent que l'école peut jouer un rôle plus important que celui de standardiser la langue ou de dire ce qui est juste ou faux, en étant un espace de réarticulation, ou mieux, un territoire pour les immondices verbales où les dichotomies du ou céderaient la place à la logique subversive du et (Lima \& Borba 2016). Par exemple, dans l'affaire de l'UFRRJ, les portes des toilettes signalaient non seulement une entrée pour les hommes «ou » pour les femmes, mais aussi une entrée pour les hétéros et les cis et les trans et les bis et les asexuellxs et les blanchxs et les noirxs, etc. De la même manière, biffer le genre grammatical à l'aide d'un $x$ effiloche le champ sémantico-pragmatique de sa portée : si, grammaticalement, les désinences o / marque du masculin et a / marque du féminin ont des référents bien circonscrits (et, donc, excluants), le $x$ embrasse tout le monde en raison de son indétermination.

Ces phénomènes indiquent que la logique binaire du ou, fondement des programmes officiels, ne parvient pas à embrasser toute la complexité de la vie sociale. Ainsi, 
l'idéologie linguistique selon laquelle " ceci est ceci, cela est cela », excluant le social de la langue, constitue aussi un trouble. Dans les pratiques quotidiennes intervenant en contexte scolaire et rendant ces lieux immondes, langue et société sont intrinsèquement liées. Comme nous avons tenté de l'argumenter ici, semer la pagaille dans les bienséantes conventions linguistiques ouvre la possibilité de «faire la différence ", de répéter la réalité de manière radicalement différente, de (ré)imaginer des futurs et de (re)dessiner des territoires de manière à ce que ces derniers ne restent pas enfermés dans des concepts immuables, puristes et bien circonscrits. Ces immondices et interventions ramènent au cœur du quotidien la puissance des ruptures, des plissements et des réinventions qui persistent à vouloir occuper la vie sociale et nous motivent ainsi à penser et agir différemment.

\section{BIBLIOGRAPHIE}

ABBOU, Julie. 2011. « Double gender marking in French: a linguistic practice of antisexism » Language Planning 12 (1) : 55-75.

ABBOU, Julie \& BAIDER, Fabienne. 2016. « Periphery, gender and language: an introduction », in Gender, language and the periphery: grammatical and social gender from the margins, ABBOU, Julie \& BAIDER, Fabienne (éds.). Amsterdam : John Benjamins, 1-24.

BAGNO, Marcos. 2013 [2011]. « O que é uma língua? Imaginário, ciência e hipóstase », in Políticas da norma e conflitos linguísticos, LAGARES, Xoán Carlos \& BAGNO, Marcos (éds.). São Paulo : Parábola, 355-387.

BAUMAN, Richard \& BRIGGS, Charles. 2003. Voices of modernity: language ideologies and the politics of inequality. Cambridge : Cambridge University Press.

BAUMAN, Zygmunt. 2010 [2003]. L'amour liquide. Paris : Fayard-Pluriel [traduit de l'anglais par Christophe Rosson].

BODINE, Ann. 1975. « Androcentrism in prescriptive grammar: singular 'they', sex-indefinite 'he', and 'he or she' " Language in Society 4 (2) : 129-146.

BORBA, Rodrigo. 2017. « The semiotic politics of affect in the Brazilian political crisis » Working Papers in Urban Language and Literacies [En ligne], (228), consulté le 17 avril 2018. URL : https:// www.academia.edu/34936078/The_semiotic_politics_of_affect_in_the_Brazilian_political_crisis BORBA, Rodrigo. 2014. « A linguagem importa? Performance, performatividade e peregrinações conceituais » Cadernos Pagu 43 : 441-474.

BOURDIEU, Pierre. 1977. «L'économie des échanges linguistiques » Langue française 34 : 17-34.

BUTLER, Judith. 2006 [1990]. Trouble dans le genre : le féminisme et la subversion de l'identité. Paris : La Découverte [traduit de l'anglais par Cynthia Kraus].

CALDAS-COULTHARD, Carmen Rosa. 2007. « Caro Colega: exclusão linguística e invisibilidade » Discurso y Sociedad 1 (2) : 230-246. 
CAMERON, Deborah. 1990. « Demythologizing sociolinguistics: Why language does not reflect society », in Ideologies of Language, JOSEPH, John \& TAYLOR, Talbot (éds.). Londres : Routledge, 79-93.

CAMERON, Deborah. 1992 [1985]. Feminism and linguistic theory. Londres : Palgrave.

CAMERON, Deborah. 2012 [1995]. Verbal hygiene. Londres : Routledge.

CAMERON, Deborah. 2014 [1991]. « Gender and language ideologies », in The handbook of Language, Gender, and Sexuality, EHRILICH, Susan, MEYERHOFF, Miriam \& HOLMES, Janet (éds.). Oxford : Wiley Blackwell, 281-296.

DERRIDA, Jacques. 1967. De la grammatologie. Paris : Éditions de Minuit.

DERRIDA, Jacques. 1972. Marges de la philosophie. Paris : Éditions de Minuit.

FARACO, Carlos Alberto. 2013 [2011]. « O Brasil entre a norma culta e a norma curta », in Políticas da norma e conflitos linguísticos, LAGARES, Xoán Carlos \& BAGNO, Marcos (éds.). São Paulo : Parábola, 259-275.

FOUCAULT, Michel. 1980. Power/knowledge: selected interviews and other writings, 1972-1977. New York : Pantheon Books.

FOUCAULT, Michel. 2010 [1976]. Histoire de la sexualité 1 : La volonté de savoir. Paris : Gallimard.

GAL, Susan. 1995. « Language, gender, and power: An anthropological review », in Gender Articulated: Language and the Socially Constructed Self, HALL, Kira \& BUCHOLTZ, Mary (éds.). Londres : Routledge, 169-182.

HALL, Kira. 2007. « X (rated) », in Encyclopedia of Sex and Gender, MALTI-DOUGLAS, Fedwa (éd.). Detroit : McMillan Reference USA, 1561-1562.

HARAWAY, Donna. 2009 [1991]. Des singes, des cyborgs et des femmes : la réinvention de la nature. Nîmes : J. Chambon \& Arles : Actes Sud [traduit de l'anglais par Oristelle Bonis].

HOUAISS, Antônio. 2001. Dicionário Houaiss de língua portuguesa. Rio de Janeiro : Autêntica.

KROSKRITY, Paul (éd.). 2004 [2000]. Regimes of languages: ideologies, polities and identities. Santa Fe : School of American Research Press.

LIMA, Fatíma \& BORBA, Rodrigo. 2016. «Sobre (des)aprendizagem e multidões queer : rizomas, multiplicidades e a política menor ", in Gênero e Diversidade Sexual: Teoria, política e educação em perspectiva, SANTOS, Douglas (éd.). Tubarão : Copiart, 79-114.

MÄDER, Guilherme \& MOURA, Heronides. 2015. « O masculino genérico sob uma perspectiva cognitivo-funcionalista » Revista do Gelne, 17 (1/2) : 33-54.

MATTOSO CÂMARA JR., Joaquim. 1975 [1972]. « Consideracões sobre o gênero em português », in Dispersos, MATTOSO CÂMARA JR., Joaquim. Rio de Janeiro : Fundação Getúlio Vargas, 115-129.

MILLER, Casey \& SWIFT, Kate. 2002 [1980]. The handbook of non-sexist writing. New York : Harper and Row.

MOITA LOPES, Luíz Paulo (éd.). 2013. O Português no século XXI: cenário geopolítico e sociolinguístico. São Paulo : Parábola.

PAUWELS, Anne. 1998. Women changing language. Londres : Longman.

PENELOPE, Julia. 1982. « Power and the opposition to feminist proposals for language change » College English 44 (8) : 840-848. 
PINTO, Joana. 2013. «Prefigurações identitárias e hierarquias linguísticas na invenção do português ", in Português na século XXI: cenário geopolítico e sociolinguístico, MOITA LOPES, Luíz Paulo (éd.). São Paulo : Parábola Editorial, 120-143.

PINTO, Joana \& BADAN, Suzana. 2012. «Feminismo e identidades no cerne dos princípios de pesquisa » Calidoscópio 10 (2) : 133-139.

SAUSSURE, Ferdinand. 1978 [1916]. Cours de linguistique générale. Paris : Payot.

SIGNORINI, Inês. 2012 [2002]. « Por uma teoria da desregulamentação linguística », in Linguística da norma, BAGNO, Marcos (éd.). São Paulo : Loyola, 85-114.

SOARES, Magda. 2012 [2002]. « Português na escola: história de uma disciplina curricular », in Linguística da norma, BAGNO, Marcos (éd.). São Paulo : Loyola, 155-178.

WETH, Constanze \& JUFFERMANS, Kasper (éds.). 2018. The tyranny of writing: Ideologies of the written word. Londres : Bloomsbury.

WOOLARD, Kathryn. A. 1998. «Introduction : Language ideology as a field of inquiry », in Language ideologies: practice and theory, SCHIEFFELIN, Bambi B., WOOLARD, Kathryn A. \& KROSKRITY, Paul (éds.). New York : Oxford University Press, 1-42.

\section{NOTES}

1. Le Colégio Pedro II accueille des élèves de la primaire au lycée [NdT].

2. Concept anglo-saxon, la littéracie, selon Jacques David, «ne correspond pas uniquement à l'alphabétisation ou à la manière de lire et d'écrire dans un système d'écriture particulier, mais doit se comprendre dans un éventail élargi de pratiques mobilisant l'écrit, possédant des finalités propres et des contextes spécifiques. L'acception s'est encore étendue, aujourd'hui, pour désigner l'ensemble des activités humaines qui recourent à l'écriture en général, mais aussi aux effets en retour de cet usage sur les modes de penser, et probablement sur l'architecture cognitive et son développement. » DAVID, Jacques. 2015. « Literacy-Litéracie-littératie : évolution et destinée d'un concept » Le français aujourd'hui 3 (190) : 9-22. URL : https://www.cairn.info/revue-le-francaisaujourd-hui-2015-3-page-9.htm [NdT].

3. Le portugais marque grammaticalement le féminin et le masculin des noms, des pronoms, des articles définis et indéfinis, des adjectifs (en attribut ou fonction de prédicat), des quantificateurs, des numéraux, des temps verbaux périphrastiques, etc. En général, le système de genre marque le féminin par la terminaison -a, par exemple a aluna (« l'écolière »), et le masculin par la terminaison -o, par exemple o aluno ( «l'écolier »). Cependant, quand il s'agit de désigner des groupes mixtes, le masculin l'emporte. Par ailleurs, le portugais comporte des mots épicènes. Tel est le cas du mot presidente, invariable dans sa forme, mais nécessitant un article qui explicite le genre du référent. Ainsi, on peut dire: o presidente («le président») ou a presidente («la présidente »).

4. Session plénière du 10 août 2016, quelques semaines avant la destitution de l'ex-présidente Dilma Rousseff. URL : https://www.youtube.com/watch?v=h9qu3vaR1EY

5. Il convient de rappeler qu'à peu d'exceptions près, les grands médias conservèrent l'usage du terme presidente pour se référer à Rousseff. Une recherche rapide dans les journaux nationaux révèle que les seuls à utiliser le terme presidenta sont politiquement marqués à gauche; ce point nécessiterait toutefois une enquête plus systématique.

6. Pour des raisons qui deviendront évidentes au fil de cet article, nous utilisons la lettre $x$ plutôt que le masculin générique. Au Brésil, l'écriture inclusive fait son apparition dans les textes académiques au début des années 2000. Parmi les stratégies visant à contourner le binarisme 
genré de la langue, l'utilisation du x est privilégiée en raison de la richesse sémiotique de cette lettre.

7. Précisons qu'en portugais, le «e » n'est la marque ni du féminin ni du masculin [NdT].

8. Lesbiennes, Gays, Bissexuels, Travestis, Transexuels, Transgenres, Queer, Intersexes

9. KAPA, Raphael. 2015. "Professores do Pedro II adotam termo "alunxs" para se referir a estudantes sem definir gênero » O Globo, consulté le 20/04/2017. URL : http://oglobo.globo.com/ sociedade/educacao/professores-do-pedro-ii-adotam-termo-alunxs-para-se-referir-estudantessem-definir-genero-17564795.

10. En nous appuyant sur Derrida (1967) et Haraway (1991), nous considérons que l'épistémè phonophallogocentrique établit le son (phono) comme l'expression de l'âme (la voix du Père), à savoir une conception forgée par des philosophes et des linguistes hommes (phallo) dans leur prétention de rationalité et d'objectivité scientifique (logos).

11. Toutefois, aux États-Unis d'Amérique, le terme Latinx, récemment devenu populaire parmi les militantxs, en lieu et place de Latino/a, se prononce $\lambda \alpha v \tau i v \varepsilon \kappa \sigma$.

12. Ici, nous utilisons délibérément le masculin générique.

13. AZEVEDO, Reinaldo. 2015. «Patrulha gayzista e feminazi chega ao Colégio Pedro II, uma instituição federal » Veja, consulté le 20/04/2017. URL : http://veja.abril.com.br/blog/reinaldo/ patrulha-gayzista-e-feminazi-chega-ao-colegio-pedro-ii-uma-instituicao-federal/

14. COLLISCHONN, Gisela \& SCHWINDT, Luiz Carlos. 2017. « Uma coisa é uma coisa; outra coisa é outra coisa. Por que a distinção entre gênero social e gramatical na língua portuguesa é necessária ao idioma » Gauchazh, consulté le 25/04/2017. URL : https://gauchazh.clicrbs.com.br/ porto-alegre/noticia/2015/12/por-que-a-distincao-entre-genero-social-e-gramatical-na-linguaportuguesa-e-necessaria-ao-idioma-4928930.html

15. L'usage du they au singulier est de nouveau considéré comme correct. Il est, de fait, devenu l'option non marquée, et cela avec l'assentiment d'institutions régulatrices comme le oxford Dictionary. Dans certains contextes informels, ce pronom remplace même les possessifs his («à lui ») et her (« à elle »). On peut dire, par exemple, John brought their computer (i.e. « John apporta son ordinateur »). En portugais, le masculin générique nécessite encore des études plus approfondies (voir, cependant, Caldas-Coulthard 2007 ; Mäder \& Moura 2015).

16. COHEN, Marina. 2015. «Reitor do Pedro II rebate críticas ao termo 'alunxs' » O Globo, consulté le 20/04/2017. URL : http://oglobo.globo.com/sociedade/educacao/reitor-do-pedro-ii-rebatecriticas-ao-termo-alunxs-17608899 Il convient de souligner que le Colégio Pedro II n'a pas institutionnalisé l'usage du $x$. L'événement qui causa tant d'émoi, i.e. l'usage du terme alunxs ("élèves ») dans l'en-tête de la copie d'examen, résulte d'un simple projet interdisciplinaire, dirigé par un professeur de biologie.

17. Pour Saussure (1978 [1916]), à la différence du signe qui serait arbitraire, « le symbole a pour caractère de n'être jamais tout à fait arbitraire; il n'est pas vide, il y a un rudiment de lien naturel entre le signifiant et le signifié » (p. 101). Cependant, la déconstruction telle que la pense Derrida (1967) nous montre comment cette distinction se base sur une métaphysique de la présence. Le philosophe et les interventions analysées dans cet article nous apprennent qu'il n'y a pas « de symbole et de signe mais un devenir signe du symbole» (p. 69), ou mieux, un symbole qui peut toujours se transformer en signifiant. 


\section{RÉSUMÉS}

En 2015, des rumeurs selon lesquelles le Colégio Pedro II $^{1}$ aurait institué l'usage du $x$ pour effacer la différence de genre (grammatical et social) motivèrent des querelles linguistiques (Cameron 2012) entre spécialistes et usagerxs ordinaires du langage. La même année, lors de la semaine d'intégration LGBT de l'UFRRJ, des affichettes collées sur les portes des toilettes et présentant des désordres linguistiques similaires provoquèrent l'indignation de certainxs étudiantxs, enseignantxs et d'une partie du personnel non enseignant. Nous partons de cette situation pour examiner ici, en dialogue avec Derrida, les discours suscités par l'effacement du genre (grammatical) dans les médias et les institutions d'enseignement, et cela dans l'objectif d'analyser les relations entre langue, société, citoyenneté et littéracie ${ }^{2}$. Nos analyses s'appuient sur le présupposé que la langue (et surtout ce que nous en faisons et ce que nous en disons) ne peut être considérée comme une question secondaire dès lors que nous prétendons appréhender des moments historiques de turbulence sociale et politique. Nous démontrerons que ces interventions sémiotiques sèment la pagaille parmi les conventions linguistiques et le quotidien des établissements scolaires et que, défiant les rapports de pouvoir, elles ouvrent la possibilité de "faire la différence », c'est-à-dire de refléter différemment la réalité, de (ré)imaginer des futurs et de (re)dessiner des territoires afin qu'ils ne restent pas enfermés dans des pratiques et des concepts immuables, puristes et bien circonscrits.

In 2015, rumours about the fact that Colégio Pedro II had established the use of the letter X as a strategy to erase (grammatical and social) gender triggered linguistic guerilla wars (Cameron, 2012) among experts and language users. In the same year, during the LGBT week at UFRRJ, signs with similar linguistic disruptions the organizers of the event had put up on the doors of the restrooms around campus provoked revolt among students, faculty members and other employees. In this scenario, in dialogue with Derrida, we investigate discourses about the erasure of (grammatical) gender in the mainstream media and in teaching institutions. Against this backdrop, we aim to analyze the relationship between language, society, citizenship, and literacies. Grounded on the analytical assumption that language (and, above all, what we do with it and speak about it) cannot not be sidelined if we want to understand historical moments of political and social turbulence, we argue that these semiotic interventions mess linguistic conventions and school's everyday life and, thus, challenge power relations. We conclude that these linguistic interventions may open up possibilities to "make a difference", i.e. to repeat reality in different ways, to (re)imagine futures and to (re)design territories in ways that liberate them from fixed, purist and well-circumscribed concepts and practices.

\section{INDEX}

Mots-clés : genre, idéologies linguistiques, littéracie, déconstruction, différance

Keywords : gender, language ideologies, literacies, deconstruction, différance

Thèmes : Recherches 


\section{AUTEURS}

\section{RODRIGO BORBA}

Professeur adjoint du Département de Lettres anglo-germaniques et du Programme interdisciplinaire de $2^{\mathrm{e}}$ et $3^{\mathrm{e}}$ cycles en linguistique appliquée à l'UFRJ

(rodrigoborba[at]letras.ufrj.br). Cet article s'inscrit dans son projet de recherche « Politiques de langue, politiques de genre : militantisme linguistique et actes de citoyenneté dans le Brésil contemporain » développé au sein de la Faculty of Linguistics, Philology, and Phonetics de l'Université d'Oxford. L'auteur remercie ici la Coordination pour le perfectionnement du personnel de l'enseignement supérieur (CAPES) qui lui a octroyé une bourse de recherche (BEX 0000061/2017-04).

\section{ADRIANA CARVALHO LOPES}

Professeure adjointe de l'Institut multidisciplinaire d'éducation à l'UFRRJ et du Programme interdisciplinaire de $2^{\mathrm{e}}$ et $3^{\mathrm{e}}$ cycles en linguistique appliquée à l'UFRJ

(adrianaclopes14[at]gmail.com). 